\title{
Comparison of Cell Morphology and Flow Cytometry in the Diagnosis of Multiple Myeloma
}

\author{
Jun Lu1, Chao Li², Yong Huang', Jiakui Zhang3* \\ ${ }^{1}$ Department of Oncology, Hefei Changhai Hospital, Hefei, China \\ ${ }^{2}$ Department of Oncology, The Second Affiliated Hospital of Anhui Medical University, Hefei, China \\ ${ }^{3}$ Department of Oncology, The Hefei Affiliated Hospital of Anhui Medical University, Hefei, China \\ Email: *6855585@sina.com
}

How to cite this paper: $\mathrm{Lu}, \mathrm{J} ., \mathrm{Li}, \mathrm{C}$., Huang, Y. and Zhang, J.K. (2020) Comparison of Cell Morphology and Flow Cytometry in the Diagnosis of Multiple Myeloma. Journal of Cancer Therapy, 11, 731-737. https://doi.org/10.4236/jct.2020.1111062

Received: October 15, 2020

Accepted: November 21, 2020

Published: November 24, 2020

Copyright $\odot 2020$ by author(s) and Scientific Research Publishing Inc. This work is licensed under the Creative Commons Attribution International License (CC BY 4.0).

http://creativecommons.org/licenses/by/4.0/ (c) (i) Open Access

\begin{abstract}
Objective: To investigate the value of cell morphology and flow cytometry in the diagnosis of Multiple myeloma (MM). Methods: 166 newly diagnosed MM patients were retrospectively analyzed, and the proportion of plasma cells detected by flow cytometry and cell morphology in these patients was statistically analyzed. Results: All patients were divided into three groups according to the proportion of plasma cells detected by cell morphology: group A ( $\geq 30 \%)$, group B ( $\geq 10 \%$ and $<30 \%)$, and group C $(<10 \%)$. 1) In all patients and group $\mathrm{A}, \mathrm{B}$ and $\mathrm{C}$, the proportion of plasma cells detected by cell morphology was all correlated with that detected by flow cytometry. 2) The proportion of plasma cells detected by cell morphology in group A and group B was higher than that by flow cytometry, while there was no significant difference in group C. 3) The sensitivity of plasma cells detected by flow cytometry in group A, B and C was $88.7 \%, 51.2 \%$ and $10 \%$, respectively. 4) According to the immunophenotypic analysis, the immunophenotypic characteristics were similar, all of them were CD19 - CD38 + CDl38 + CD56 +/- CD45dim Conclusion: There is a certain correlation between the two detection methods. The quantitative detection of myeloma cells by cell morphology is better, and the qualitative detection by flow cytometry is better. Combined with the two detection methods, the detection rate of multiple myeloma can be significantly improved.
\end{abstract}

\section{Keywords}

Multiple Myeloma, Cell Morphology, Flow Cytometry, Immune Classification 


\section{Introduction}

Multiple myeloma (MM) is a plasma cell malignant clonal disease, accounting for $1 \%$ of all tumors and $10 \%$ of hematological tumors [1]. It is the second most common hematological tumor. The main feature of MM is malignant proliferation of monoclonal plasma cells in bone marrow accompanied by extensive infiltration, including bone, nervous system, liver, spleen, lymph nodes, etc. At present, the laboratory testing of MM includes bone marrow morphology, bone marrow biopsy, immunofixation electrophoresis, flow cytometry, chest CT, $\mathrm{X}$-ray, fluorescence in situ hybridization (FISH), etc. Bone marrow cytology is important in the diagnosis of $\mathrm{MM}$, but the proportion of plasma cells may not meet the diagnostic criteria for the focal distribution characteristics of myeloma cells [2]. Moreover, well differentiated myeloma cells are difficult to distinguish with normal plasma cells. In recent years, the rapid development of flow cytometry provides an important basis for improving the detection of mm, which is more rapidly, objectively and sensitively. At the same time, it can detect the minimal residual lesions (MRD) and provide the basis for the prognosis [3].

In clinical, the proportion of leukemia cells detected by flow cytometry in some types of leukemia such as acute lymphocytic leukemia (ALL) and acute myeloid leukemia (AML) is always more than that of bone marrow cytology, but the detection of MM is opposite [4]. Therefore, to analyze the correlation and comparison of cell morphology and flow cytometry is useful to improve the diagnostic rate of MM.

\section{Materials and Methods}

We retrospective analyzed $166 \mathrm{MM}$ patients diagnosed in the Second Affiliated Hospital of Anhui Medical University from October 2013 to June 2018. The diagnosis was in accordance with the International Myeloma Working Group updated criteria for the diagnosis of multiple myeloma [5]. Among $166 \mathrm{MM}$ patients, 98 were male and 68 were female. The age of diagnosis was $32-96$ years old with an average age of $65.11 \pm 10.86$ years. Among 166 patients, the clinical manifestations were different, including 108 cases of bone destruction (65.0\%), 101 cases of anemia (60.8\%), 121 cases of bone pain (72.9\%), 86 cases of renal function damage (29.5\%), 11 cases of bleeding (10.8\%), 1 case of hyperviscosity (0.6\%). According to the immunoglobulin classification, there were 82 cases of IgG type, 48 cases of IgA type, 2 cases of IgD type, 14 cases of $\kappa$ type, 19 cases of $\lambda$ type and 1 case of non secretory type. According to the international staging system (ISS), there were 11 cases in stage I, 52 cases in stage II and 103 cases in stage III. The results of cell morphology and flow cytometry in 166 patients were diagnosed and verified by two physicians (one of whom was the chief physician).

In order to compare the value of cell morphology with flow cytometry in the diagnosis of $\mathrm{MM}$, we divided all patients into three groups according to the proportion of plasma cells detected by cell morphology, including group A $(\geq 30 \%, n=93)$, group $B(\geq 10 \%$ and $<30 \%, n=43)$, group $C(<10 \%, n=30)$. 
FITC markers of IgG1, CD19, CD13, HLA-DR, CD64, CD8, CD5, CD14, CD4, CD20, CD45 monoclonal antibodies. PE marked IgG1, CD56, CD13, CD33, CD34, CD3, CD10, CD40, CD25, CD7, CD49e monoclonal antibodies. PE-Cy5 mark CD8 monoclonal antibodies. Standard, red blood cell cracking liquid fluorescent microspheres are bought from Beckman-Counlter companies in the United States.

\section{Statistical Analysis}

SPSS 19.0 statistical software was used for data analysis. The data conforming to normal distribution were represented by, and those that did not conform to normal distribution were expressed by quartile interval. Rank correlation analysis was used to analyze the proportion of MM plasma cells obtained by two different detection methods. Rank sum test was used to compare the measurement data within and between groups, and the difference was statistically significant if $\mathrm{p}$ value was less than 0.05 .

\section{Results}

\subsection{Correlation between Cytological Morphology and Flow Cytometry in the Detection of Plasma Cells}

In all patients, the proportion of plasma cells detected by cell morphology was correlated with that detected by flow cytometry $(\mathrm{r}=0.748, \mathrm{P}<0.001)$. In group A, B and C, there was a correlation between the two methods $(r=0.496,0.477$, $0.566, \mathrm{P}<0.05)$. Among them, 136 cases $(81.9 \%)$ of plasma cells were detected by cell morphology, and 107 cases $(64.5 \%)$ were detected by flow cytometry.

\subsection{Differences between Cytological Morphology and Flow Cytometry in the Detection of Plasma Cells}

In all patients, the proportion of myeloma cells in bone marrow cytology was higher than that in flow cytometry $(\mathrm{P}<0.001)$. In group $\mathrm{A}$ and $\mathrm{B}$, the proportion of plasma cells detected by bone marrow flow cytometry was higher than that by flow cytometry $(\mathrm{P}<0.001)$. There was no significant difference between the two methods in group C (See Table 1).

\subsection{Sensitivity of Flow Cytometry in the Diagnosis of MM}

In group $\mathrm{A}, \mathrm{B}$ and group $\mathrm{C}$, the proportion of plasma cells detected by flow cytometry met the diagnostic criteria of $\geq 10 \%$ in 82 cases, 22 cases and 3 cases, respectively. The sensitivity of diagnosis of $\mathrm{mm}$ was $88.7 \%, 51.2 \%$ and $10 \%$ respectively.

\subsection{Immunophenotype of Plasma Cell in All Patients Detected by Flow Cytometry}

The immunophenotype was CD38 + CD138 + CD19 - CD56 - CD45dim - in 31 patients, CD38 + CD138 + CD19 - CD56 + CD45dim - in 128 patients. Among all patients, CD19 was all negative. The positive rate of CD38, CD138, CD56, CD45 was 100\%, 100\%, 80.7\%, and 3.0\%, respectively (See Table 2). 
Table 1. Detection of two methods in the detection of plasma cells proportion. Results are provided as median (25th to 75 th percentile).

\begin{tabular}{ccccc}
\hline Type & All patients (\%) & Group A (\%) & Group B (\%) & Group C (\%) \\
\hline The cell morphology & $40.5(36-64)$ & $55.5(43-68)$ & $20.5(16-26)$ & $12.5(4.0-18.0)$ \\
Flow cytometry & $30.5(21-53)$ & $38.5(29.6-55.1)$ & $14.5(10.6-23.0)$ & $8.5(4.0-18.0)$ \\
P & $<0.001$ & $<0.001$ & $<0.001$ & $>0.05$ \\
\hline
\end{tabular}

Table 2. Immunophenotype of plasma cells in multiple myeloma.

\begin{tabular}{cccc}
\hline Fluid-flow antigens & Positive cases (n) & $\begin{array}{c}\text { The total number of } \\
\text { cases (n) }\end{array}$ & Positive rate (\%) \\
\hline CD19 & 2 & 166 & 1.2 \\
CD56 & 134 & 166 & 80.7 \\
CD38 & 166 & 166 & 100 \\
CD138 & 166 & 166 & 100 \\
CD45 & 5 & 166 & 3.0 \\
\hline
\end{tabular}

\section{Discussion}

$\mathrm{MM}$ is a plasma cell malignant tumor with slow progression even several years. The clinical manifestations of MM are complex, with different onset and lack of specificity. So it is often misdiagnosed as other corresponding common diseases. Therefore, early diagnosis of MM is of great significance to improve the prognosis. Due to the different experience of different doctors, the master scale of diagnostic criteria and diagnosis and treatment level are not the same and the clinical misdiagnosis rate is high [6]. In our study, the results showed that $81.9 \%$ MM patients had more than $10 \%$ of plasma cells, while only $64.5 \%$ than that detected by flow cytometry. It is suggested that bone marrow cytology is of great significance in the diagnosis of MM, and it is the main means in the diagnosis of MM. The diagnostic criteria of MM take the detection of monoclonal plasma cells by bone marrow cytology as the first diagnostic standard, which fully reflects the diagnostic value of bone marrow cytology in MM.

Our study showed that there was a high correlation between cell morphology and flow cytometry in the detection of plasma cell proportion. Cell morphology was better than flow cytometry in detecting the proportion of plasma cells. Report of Rawstron AC, et al. is consistent with our results. The possible reasons may be related to the dilution of bone marrow fluid, the existence of plasma cell clusters, and the fragility of plasma cells during sample preparation [4].

In addition to the diffuse infiltration, the monoclonal plasma cells in MM also showed focal distribution. With the deepening understanding of MM in recent years, the diagnosis of MM has been gradually improved. In our study, the proportion of plasma cells detected by bone marrow cytology in 30 patients is still 
less than $10 \%$. The final diagnosis was made by bone marrow pathology, hematuria immunofixation electrophoresis, and related target organ damage. We also performed flow cytometry in these patients, and found that the immunophenotype of these 30 patients was similar. Referring to the relevant literature, it was consistent with the diagnosis of MM [4].

In recent years, flow cytometry (FCM) has become an important method for the diagnosis of MM due to the small sample size required for detection of plasma cells [7]. Plasma cells are the terminal stage of B cell differentiation. The loss of B cell line specific antigen and surface immunoglobulin makes plasma cell surface antigen heterogeneous. The membrane antigen molecules of myeloma cells also have great heterogeneity with normal plasma cells, such as CD19, CD27, CD38, CD45, CD56, CD138, etc. At present, it was found that most MM cells expressed CD38 + CD138 + CD56 + CD19 - CD45 - or weakly positive, which was consistent with the results of this study [8]. The differentiation of benign and malignant plasma cells mainly depends on monoclonal plasma cell immunophenotype CD138 + CD38 + CD19 - CD56 + and normal plasma cell phenotype CD138 + CD38 + CD19 + CD56 -.

CD38 and CD138 were highly expressed in almost all plasma cells [8]. Compared with myeloma cells, ordinary plasma cells expressed stronger CD38 and weaker CD138. Studies have shown that the loss of CD138 antigen on tumor cell surface is closely related to the prognosis of MM patients [9]. CD56 is a surface antigen of NK cells, which can hardly be detected in normal mature plasma cells, but expressed in most monoclonal cells. CD56 was not expressed in circulating plasma cells and extramedullary myeloma. Some studies have suggested that the loss of CD56 expression in myeloma cells is related to extramedullary infiltration, suggesting a poor prognosis [10]. Normal plasma cells expressed B cell antigen CD19, while myeloma cells hardly expressed CD19. Immature plasma cells expressed high levels of CD45, and the expression of CD45 gradually decreased with the differentiation of plasma cells, and the clonal plasma cells in myeloma showed heterogeneous expression [11]. Some studies have suggested that the proportion of CD45 + plasma cells in the early stage of plasma cell proliferation, such as unknown monoclonal immunoglobulin disease and smoldering multiple myeloma (SMM), is significantly higher than that of newly diagnosed and relapsed MM [12]. Therefore, CD38 ++ CD138 + CD45 + CD19 + CD56 - and $\mathrm{CD} 38++\mathrm{CD} 138+\mathrm{CD} 45-\mathrm{CD} 19-\mathrm{CD} 56+/-$ are the main immunophenotypes to differentiate benign and malignant plasma cells.

In conclusion, bone marrow cytology is superior to flow cytometry in detecting the proportion of plasma cells in the diagnosis of $\mathrm{mm}$, and flow cytometry can analyze the nature of plasma cells by immunophenotyping, guide the treatment, judge the prognosis and monitor MRD. The detection rate of MM can be improved by combining the two methods. The present date may be a reconfirmation of the pre-existing date, however, considering the importance of MM diagnosis and treatment, the present date may contribute to better handling MM 
in this area/hospital.

\section{Conflicts of Interest}

The authors declared that they have no competing interests.

\section{Funding}

This work is funded by the Key research and development projects in Anhui Province (Project No: 201904a07020032). The authors thank the patient and his family and all the investigators, including the physicians and laboratory technicians in this study.

\section{References}

[1] Brigle, K. and Rogers, B. (2017) Pathobiology and Diagnosis of Multiple Myeloma. Seminars in Oncology Nursing, 33, 225-236. https://doi.org/10.1016/j.soncn.2017.05.012

[2] Vincent Rajkumar, S. (2019) Multiple Myeloma: Every Year a New Standard? Hematological Oncology, 37, 62-65. https://doi.org/10.1002/hon.2586

[3] Roshal, M. (2018) Minimal Residual Disease Detection by Flow Cytometry in Multiple Myeloma: Why and How? Seminars in Hematology, 55, 4-12. https://doi.org/10.1053/j.seminhematol.2018.02.011

[4] Rawstron, A.C., Orfao, A., Beksac, M., et al. (2008) Report of the European Myeloma Network on Multiparametric Flow Cytometry in Multiple Myeloma and Related Disorders. Haematologica, 93, 431-438. https://doi.org/10.3324/haematol.11080

[5] Rajkumar, S.V., Dimopoulos, M.A., Palumbo, A., et al. (2014) International Myeloma Working Group Updated Criteria for the Diagnosis of Multiple Myeloma. The Lancet Oncology, 15, E538-E548. https://doi.org/10.1016/S1470-2045(14)70442-5

[6] Joshua, D.E., Bryant, C., Dix, C., et al. (2019) Biology and Therapy of Multiple Myeloma. Medical Journal of Australia, 210, 375-380.

https://doi.org/10.5694/mja2.50129

[7] Bao, Z.J. and Liu, X. (2016) Research Progress on Role of Flow Cytometry in Diagnosis of Multiple Myeloma-Review. Journal of Experimental Hematology, 24, 1611-1614.

[8] Bataille, R., Jégo, G., et al. (2006) The Phenotype of Normal, Reactive and Malignant Plasma Cells. Identification of "Many and Multiple Myelomas" and of New Targets for Myeloma Therapy. Haematologica, 91, 1234-1240.

[9] Lovell, R., Dunn, J.A., et al. (2005) Soluble Syndecan-1 Level at Diagnosis Is an Independent Prognostic Factor in Multiple Myeloma and the Extent of Fall from Diagnosis to Plateau Predicts for Overall Survival. British Journal of Haematology, 130, 542-548. https://doi.org/10.1111/j.1365-2141.2005.05647.x

[10] Blaheta, R.A., Beecken, W.-D., Engl, T., et al. (2004) Human Cytomegalovirus Infection of Tumor Cells Downregulates NCAM (CD56): A Novel Mechanism for Virus-Induced Tumor Invasiveness. Neoplasia, 6, 323-331. https://doi.org/10.1593/neo.03418

[11] An, G., Xu, Y., Shi, L.H., et al. (2013) t(11;14) Multiple Myeloma: A Subtype Associated with Distinct Immunological Features, Immunophenotypic Characteristics but Divergent Outcome. Leukemia Research, 37, 1251-1257. https://doi.org/10.1016/j.leukres.2013.06.020 
[12] Kumar, S., Rajkumar, S.V., Kimlinger, T., et al. (2005) CD45 Expression by Bone Marrow Plasma Cells in Multiple Myeloma: Clinical and Biological Correlations. Leukemia, 19, 1466-1470. https://doi.org/10.1038/sj.leu.2403823 